\title{
EFFECT OF VANCOMYCIN THERAPY FOR OSTEOMYELITIS on Colonization by Methicillin-Resistant STAPHYLOCOCCUS AUREUS: LACK OF EMERGENCE OF GLYCOPEPTIDE RESISTANCE
}

\author{
Louis Bernard, MD; Pierre Vaudaux, PhD; Albert Vuagnat, MD; Richard Stern, MD; Peter Rohner, MD; Didier Pittet, MD, MS; \\ Jacques Schrenzel, MD; Pierre Hoffmeyer, MD; Osteomyelitis Study Group
}

\begin{abstract}
BACKGROUND: In treating orthopedic infections, the long-term impact of vancomycin therapy on colonization by methicillin-resistant Staphylococcus aureus (MRSA) and the emergence of vancomycin-intermediate $S$. aureus is unknown.

DESIGN: Prospective surveillance of the effect of longterm vancomycin therapy on colonization by MRSA and the emergence of vancomycin-intermediate $S$. aureus.

METHODS: Thirty-four patients with MRSA osteomyelitis that was microbiologically documented were longitudinally observed for the emergence of vancomycin-intermediate $S$. aureus at 3 body sites (wound, anterior nares, and groin) during the initial period of vancomycin therapy and at the 2-month follow-up. Twenty patients received the standard dose (20
\end{abstract}

The prevention and treatment of methicillin-resistant Staphylococcus aureus (MRSA) infections represent an increasingly difficult challenge due to the emergence of multidrug-resistant strains. An additional concern is the recent discovery of MRSA isolates that exhibit decreased susceptibility to glycopeptides, including vancomycin, ${ }^{1-4}$ which raises the possibility of organisms exhibiting global resistance to all currently available antibiotics. ${ }^{5}$ These isolates may demonstrate either intermediate susceptibility or resistance to vancomycin. ${ }^{6}$ Detection of vancomycin resistance is particularly difficult in most clinical isolates determined as either heterogeneous vancomycin-intermediate $S$. aureus (VISA) ${ }^{2}$ or heterogeneous vancomycin-resistant $S$. aureus (VRSA).$^{1-3}$

Several clinical and epidemiologic reports indicate that nasal and extranasal carriage of MRSA may predispose targeted groups of patients to invasive infections and contribute to the nosocomial dissemination of these dangerous pathogens. ${ }^{7}$ Because patients with osteomyelitis caused by MRSA usually require several weeks of parenteral administration of vancomycin, they should theoretically be at risk for $\mathrm{mg} / \mathrm{kg} / \mathrm{d}$ ) for $34 \pm 6$ days and 14 patients received a high dose ( $40 \mathrm{mg} / \mathrm{kg} / \mathrm{d}$ ) of vancomycin for $37 \pm 9$ days.

RESULTS: During vancomycin treatment, global MRSA carriage (all body sites) fell from 100\% to $25 \%$ in the group of patients receiving the standard dose of vancomycin, and from $100 \%$ to $40 \%$ in the group receiving the high dose. During the $2-$ month follow-up period after vancomycin therapy, global MRSA carriage increased from $25 \%$ to $55 \%$ in the group receiving the standard dose and decreased from $43 \%$ to $36 \%$ in the group receiving the high dose.

CONCLUSION: Therapy with a high dose of vancomycin contributes to the sustained eradication of MRSA carriage without promoting the emergence of glycopeptide resistance (Infect Control Hosp Epidemiol 2003;24:650-654).

the emergence of glycopeptide-resistant subpopulations. ${ }^{810}$ Paradoxically, there has been no study evaluating the impact of long-term vancomycin therapy on the risk of emergence of VISA or VRSA in either infected or colonized body sites of patients treated for MRSA osteomyelitis. Although the use of mupirocin for topical MRSA decontamination has shown some efficacy for prophylaxis of orthopedic infections, ${ }^{11}$ its impact on multiple carriage sites of patients treated for MRSA osteomyelitis has not been reported.

The aim of this study was to evaluate the influence of prolonged vancomycin therapy for MRSA osteomyelitis on the persistence of MRSA carriage and the potential emergence of VISA or VRSA in sequentially cultured body sites during the first 4-week period of antimicrobial therapy and at the 2-month follow-up.

\section{METHODS}

\section{Population and Study Design}

Between October 1998 and September 2001, we enrolled 39 adult patients consecutively admitted to a specifically designed orthopedic septic unit at the University

Drs. Bernard, Stern, and Hoffmeyer are from the Orthopedic Clinic; Drs. Vaudaux, Rohner, Schrenzel, and Bernard are from the Division of Infectious Diseases; and Dr. Pittet is from the Infection Control Program, Geneva University Hospital, Geneva, Switzerland. Dr. Vuagnat is from the Department of Statistics, St. Michel Hospital, Angoulème, France.

Address reprint requests to Dr. Louis Bernard, Orthopedic Clinic, Geneva University Hospital, 24 rue Micheli-du-Crest, 1211 Geneva 14, Switzerland.

Supported in part by grants 20010105 (LB), 623-57 950.99 (JS), and $132-63710.00$ (PV) from the Synos Foundation; and grant 4049063250/1 from the Swiss National Foundation for Scientific Research.

The authors thank all members of the septic orthopedic surgery unit and members of the Infection Control Program and the Clinical Microbiology Laboratory for their support and assistance. The authors also thank the surgeons of the orthopedic clinic for including their patients in this study, E. Huggler for technical assistance, and S. Dharan for MRSA genotyping. 
Hospital of Geneva. The criteria for eligibility included microbiologically proven osteomyelitis with a pure culture of MRSA from a bone biopsy and intravenous vancomycin therapy for a minimum of 4 weeks. Five patients were excluded due to incomplete vancomycin therapy, allergy to vancomycin, or incomplete microbiological data.

The first subgroup of enrolled patients (from October 1998 to November 2000) were treated intravenously with a standard dose $(20 \mathrm{mg} / \mathrm{kg} / \mathrm{d})$ of vancomycin (Eli Lilly, Geneva, Switzerland), whereas the second subgroup of enrolled patients (from December 2000 to December 2001) received a high dose $(40 \mathrm{mg} / \mathrm{kg} / \mathrm{d})$ of intravenous vancomycin. The treatment was then adjusted to obtain a trough vancomycin concentration in the serum of 20 to $25 \mathrm{mg} / \mathrm{L}$ in the group receiving the high dose, or 10 to $15 \mathrm{mg} / \mathrm{L}$ in the group receiving the standard dose, and to have a peak less than $50 \mathrm{mg} / \mathrm{L}$. Thereafter, samples were taken only weekly or if an increase in serum creatinine was noted.

During the first week of vancomycin therapy, all patients received applications of $4 \%$ chlorhexidine soap (Hibiscrub, Zeneca Pharma, Cergy, France) for daily body cleansing and $2 \%$ intranasal calcium mupirocin (Bactroban Nasal, SmithKline Beecham, Thörishaus, Switzerland) for 5 days. In addition, each patient remaining a MRSA carrier at any body site (wound, nose, or groin) during hospitalization was placed in contact isolation according to the established guidelines of our institution $^{12}$ until eradication was assessed by 2 consecutive negative surveillance cultures.

\section{Microbiologic Evaluation}

All patients were screened for MRSA immediately before starting vancomycin therapy. Swabs (Copanswabs, Copan Italia S.p.A., Brescia, Italy) were taken from the anterior nares, the groin, and any wound, if present.

Qualitative cultures were performed after enrichment in Staphylococcus Broth $\mathrm{NaCl}$ (Difco Laboratories, Sparks, MD) for 24 hours and subculturing on sheep blood agar. Identification of $S$. aureus was based on the morphology of colonies, the presence of clumping factor or protein A (Pastorex, Bio-Rad, Steinach, Switzerland), and the production of heat-stable nuclease (Bacto DNase test agar [Difco Laboratories] and toluidine blue O [Merck, Darmstadt, Germany]). Each MRSA isolate was routinely monitored for its overall antimicrobial susceptibility by disk diffusion (Sirscan, v2a, Montpellier, France) according to National Committee for Clinical Laboratory Standards guidelines, ${ }^{13}$ and finally stored at $-70^{\circ} \mathrm{C}$. Follow-up screening cultures for MRSA were performed weekly in all 3 sites during the initial 4-week period of therapy and at least once during the 2month follow-up period. The eradication of MRSA was defined by at least 3 negative culture sets from body sites.

\section{Susceptibility Testing}

From each patient in whom MRSA was not eradicated during vancomycin therapy, 1 pre-therapy, 1 on-therapy (at 3 to 4 weeks), and 1 post-therapy isolate were screened for the presence of subpopulations growing on brain-heart infusion agar supplemented with $2 \mu \mathrm{g} / \mathrm{mL}$ of vancomycin. One hundred microliters of a bacterial suspension adjusted to the McFarland Standard Indicator 2.0 (ATB 1550, API bioMérieux, Marcy l'Etiole, France) (approximately $5 \times 10^{8}$ colony-forming units $[\mathrm{CFU}] / \mathrm{mL}$ ) was spread onto vancomycin brain-heart infusion agar. $S$. aureus strains American Type Culture Collection 29213 and mu50 were used as the negative and positive quality control strains, respectively. ${ }^{2}$ Any colony recovered from the agar at 48 hours was further tested for vancomycin minimum inhibitory concentration (MIC) by the Etest.

All strains of MRSA were also tested for the potential presence of VISA or heterogeneous VISA by the Etest (AB BIODISK, Solna, Sweden) using the McFarland 2.0 inoculum. ${ }^{14}$ VISA isolates were defined as having a vancomycin MIC ranging between 4 and $16 \mu \mathrm{g} / \mathrm{mL}$, according to the breakpoints of the French Microbiology Society. ${ }^{15}$ The VISA control was the mu50 strain.

\section{Strain Typing}

Epidemiologic typing of sequential MRSA isolates was performed by pulsed-field gel electrophoresis (PFGE) (Pulsaphor system, Pharmacia LKB Biotechnology, Uppsala, Sweden) of chromosomal DNA digested with restriction endonuclease SmaI (Bio-Rad, Hercules, CA), according to established protocols. ${ }^{16}$ The clonality of MRSA-persistent isolates was evaluated on specimens collected at baseline, on-therapy (at 3 to 4 weeks), and during follow-up. Clonal diversity was defined by Tenover et al. ${ }^{17}$

\section{Therapeutic Regimens and Assessment of Serum Levels of Vancomycin}

Peak or trough serum levels of vancomycin were measured with the TDX Analyzer (Abbott Laboratories, Vienna, Austria) at 30 minutes following the 1-hour infusion or immediately before the next infusion, respectively.

\section{Data Analysis}

In this study, the demographic and clinical information that was recorded included age and gender, underlying diseases, type and stage of osteomyelitis, type of vancomycin regimen, serum levels of vancomycin, and other associated antibiotics. Continuous variables were presented as the mean and standard deviation or median if their distribution was skewed. Yates-corrected chi-square or Fisher's exact tests were used to assess changes between the observed proportions of unrelated samples. A $P$ value of less than .05 was considered statistically significant. Statistical analysis was performed using Analyse-it software (version 1.62; Analyse-it Software LTD, Leeds, England) for Excel 2002 (Microsoft Corp., Redmond, WA).

\section{RESULTS}

\section{Patients and Vancomycin Treatment}

Thirty-four patients ( 20 men and 14 women) with a mean age of $66.2 \pm 16.1$ years (range, 37 to 77 years) treat- 
TABLE

Characteristics of Orthopedic Patients Treated With VANCOMYCIN

\begin{tabular}{|c|c|c|}
\hline \multirow[b]{2}{*}{ Characteristic } & \multicolumn{2}{|c|}{ Vancomycin Regimen } \\
\hline & $\begin{array}{l}\text { Standard Dose } \\
(20 \mathrm{mg} / \mathrm{kg} / \mathrm{d})\end{array}$ & $\begin{array}{l}\text { Hlgh Dose } \\
(40 \mathrm{mg} / \mathrm{kg} / \mathrm{d})\end{array}$ \\
\hline No. of patients & 20 & 14 \\
\hline Mean age, y $( \pm \mathrm{SD})$ & $67( \pm 18)$ & $66( \pm 12)$ \\
\hline Male (\%) & $10(50)$ & $8(57)$ \\
\hline \multicolumn{3}{|l|}{ No. with underlying diseases (\%) } \\
\hline Chronic renal failure & $4(20)$ & $4(29)$ \\
\hline Diabetic disease & $6(30)$ & $7(50)$ \\
\hline \multicolumn{3}{|l|}{ No. with osteomyelitis (\%) } \\
\hline Cierny-Mader, stage 3 or $4^{*}$ & $7(35)$ & $6(43)$ \\
\hline \multicolumn{3}{|l|}{ Localization of osteomyelitis } \\
\hline Foot-phalangeal & $9(45)$ & $7(50)$ \\
\hline Femur-hip & $4(20)$ & $3(21)$ \\
\hline Tibia-knee & $4(20)$ & $3(21)$ \\
\hline Other $^{\dagger}$ & $3(15)$ & $1(8)$ \\
\hline Implant-associated infection (\%) & $4(20)$ & $4(29)$ \\
\hline $\begin{array}{l}\text { No. who underwent surgical } \\
\text { procedures }(\%)\end{array}$ & $17(84)$ & $11(79)$ \\
\hline \multicolumn{3}{|l|}{ Vancomycin regimen } \\
\hline $\begin{array}{l}\text { Mean trough serum level, } \\
m g / L( \pm S D)\end{array}$ & $11.4( \pm 7.1)$ & $21.8( \pm 5.9)$ \\
\hline $\begin{array}{l}\text { Mean duration of treatment, } \\
d( \pm \text { SD) }\end{array}$ & $34( \pm 6)$ & $37( \pm 9)$ \\
\hline $\begin{array}{l}\text { No. of patients with other } \\
\text { antibiotics }(\%)^{\ddagger}\end{array}$ & $5(25)$ & $4(29)$ \\
\hline
\end{tabular}

$\mathrm{SD}=$ standard deviation.

*Cierny-Mader classification: stage $\mathbf{1}$ = intramedullary osteomyelitis (infected intramedullary rod); stage 2 - superficial osteomyelitis; stage 3 = localized osteomyelitis (full-thickness, cortical sequestration); and stage 4 = diffuse osteomyelitis (fracture with non-union, infected prosthesis) 'Included ankle, wrist, or phalangeal.

Rifampin and ciprofloxacin were used in combination with vancomycin in 8 patients and 1 patient, respectively.

ed for MRSA osteomyelitis with vancomycin for a minimum of 4 weeks fulfilled all criteria to be enrolled in this study. There was no significant difference in the major baseline demographic and clinical characteristics of patients treated with the standard $(n=20)$ or high $(n=14)$ dose of vancomycin, including age, gender, duration of therapy, implant-associated infection, and major underlying diseases (Table). The localization and severity of osteomyelitis was similar in patients treated with the standard dose or the high dose of vancomycin. Nine patients (4 in the group receiving the standard dose and 5 in the group receiving the high dose) were diagnosed as having an acute osteomyelitis, whereas 25 patients suffered from a chronic infection (duration, longer than 4 weeks). Twenty-six patients (14 receiving the standard dose and 12 receiving the high dose) initially presented with an open wound (chronic fistula or breakdown of a wound following surgery).

Twenty patients received a standard dose (20 $\mathrm{mg} / \mathrm{kg} / \mathrm{d})$ and 14 patients a high dose $(40 \mathrm{mg} / \mathrm{kg} / \mathrm{d})$ of vancomycin for $34 \pm 6$ and $37 \pm 9$ days, respectively. Serum levels of vancomycin were used for adjusting the standard- or high-dose regimens of intravenous vancomycin to target trough serum levels of 10 to $15 \mathrm{mg} / \mathrm{L}$ and 20 to 25 $\mathrm{mg} / \mathrm{L}$, respectively. The mean ( \pm standard deviation) serum trough levels in patients receiving the standard and high doses of vancomycin were $11.4 \pm 7.1 \mathrm{mg} / \mathrm{L}$ and $21.8 \pm 5.9 \mathrm{mg} / \mathrm{L}$, respectively. Eight patients received another systemic antibiotic combined with vancomycin; 3 receiving the standard dose and 4 receiving the high dose, respectively, received rifampin and 1 patient receiving a high dose received ciprofloxacin.

\section{Rate of MRSA Colonization During and After Glycopeptide Therapy}

At baseline, $100 \%$ of the patients had MRSA osteomyelitis that was microbiologically documented (by bone biopsy). In the group receiving the standard dose of vancomycin, 9 (45\%) of the 20 patients were colonized in the anterior nares and $6(30 \%)$ in the groin. In comparison, at the onset of therapy with a high dose of vancomycin, 10 (71\%) of the 14 patients were colonized in the nares and 9 (64\%) in the groin.

During vancomycin treatment, MRSA nasal carriage fell from $45 \%$ to $5 \%$ in the group receiving the standard dose of vancomycin, and from $71 \%$ to $14 \%$ in the group receiving the high dose (no significant difference). MRSA groin carriage fell from $30 \%$ to $10 \%$ in the standard-dose group, and from $64 \%$ to $21 \%$ in the high-dose group (no significant difference). The global MRSA carriage (all body sites) fell from $100 \%$ to $25 \%$ in the standard-dose group, and from $100 \%$ to $40 \%$ in the high-dose group (no significant difference). During the 2-month period following therapy with the standard dose of vancomycin, 6 of 15 previously decolonized patients were recolonized with MRSA, shifting the overall colonization rate for this period from $25 \%$ to $55 \%$.

During the 2-month follow-up period after therapy with a high dose of vancomycin, overall MRSA carriage at any site did not increase, but marginally decreased from $43 \%$ to $36 \%$ (Figure). Patients treated with a high dose of vancomycin had a significantly higher probability $(P=$ .048) of remaining free of MRSA during the follow-up period than did patients treated with the standard dose.

An analysis of risk factors showed that among the residual MRSA carriers treated with vancomycin, most still had open wounds. Except for the vancomycin dose, no factors (eg, underlying diseases, implant-associated infection, or other associated antibiotics) should be associated with a risk of residual MRSA carriage.

\section{Genetic Relatedness of MRSA Specimens}

In the 16 patients who were persistently or recurrently colonized with MRSA during follow-up screening, PFGE was used to evaluate the genetic relatedness of pretreatment and follow-up strains. In all 16 patients, 11 who received the standard dose and 5 who received the high dose, the relatedness of persistently or recurrently colo- 
nizing strains of MRSA was confirmed and no patient became recolonized with a new strain.

\section{Analysis of Vancomycin Susceptibility}

The potential emergence of VISA or heterogeneous VISA was determined by comparing follow-up isolates with related pretreatment isolates in the 16 persistent or recurrent MRSA carriers. Among the 51 MRSA isolates tested, none yielded any significant subpopulation (more than 10 $\mathrm{CFU}$ ) that demonstrated decreased vancomycin susceptibility on agar supplemented with $2 \mu \mathrm{g} / \mathrm{mL}$ of vancomycin. The screening of subpopulations on brain-heart infusion agar supplemented with $2 \mu \mathrm{g} / \mathrm{mL}$ of vancomycin was used to avoid extensive population analysis studies on all MRSA isolates. For the few MRSA isolates that yielded colonies growing on agar containing $2 \mu \mathrm{g} / \mathrm{mL}$ of vancomycin, further testing demonstrated that none of these colonies could grow on agar supplemented with higher concentrations (ie, 4 or $6 \mu \mathrm{g} / \mathrm{mL}$ ) of vancomycin, nor did any exhibit a significant increase in vancomycin MIC by the Etest.

Evaluation of the $51 \mathrm{MRSA}$ isolates by the Etest yielded identical MICs (median MIC, $1.5 \mu \mathrm{g} / \mathrm{mL}$; range, 1 to $2 \mu \mathrm{g} / \mathrm{mL}$ ) for follow-up MRSA isolates from 11 carriers treated with the standard dose of vancomycin and 5 carriers treated with the high dose. In no patient was there any trend for the emergence of VISA or heterogeneous VISA during the long-term intravenous therapy with either the standard or high dose.

\section{DISCUSSION}

This study evaluated the impact of long-term parenteral vancomycin therapy on MRSA carriage or eradication at 3 body sites and the potential emergence of vancomycin resistance in hospitalized orthopedic patients treated for osteomyelitis. The 2 groups of patients treated with vancomycin received a similar 1-week protocol of topical decontamination with mupirocin and chlorhexidine, but differed from each other by receiving either a standard or high dose of parenteral vancomycin. Whereas eradication of MRSA carriage occurred in $75 \%$ and $57 \%$ of the patients treated for 4 weeks with the standard or high dose of vancomycin, respectively, recolonization observed during the 2-month post-therapy period occurred mainly in the group receiving the standard dose. Decolonization of the nares essentially occurred during the first week of therapy. Overall, these data indicate the efficacy of mupirocin applied intranasally. But this efficacy is temporary because previously decolonized patients in the group receiving the standard dose of vancomycin were recolonized with MRSA during the fourth week and 2-month period of follow-up. However, no recolonization occurred among patients in the group receiving the high dose. All persisting or relapsing MRSA isolates were identical to pre-therapy isolates and remained fully susceptible to vancomycin during the observation period, regardless of the serum levels of vancomycin obtained during therapy or duration of antibiotic exposure.

Whereas many studies have been performed to evaluate the efficacy of topical or systemic antimicrobial

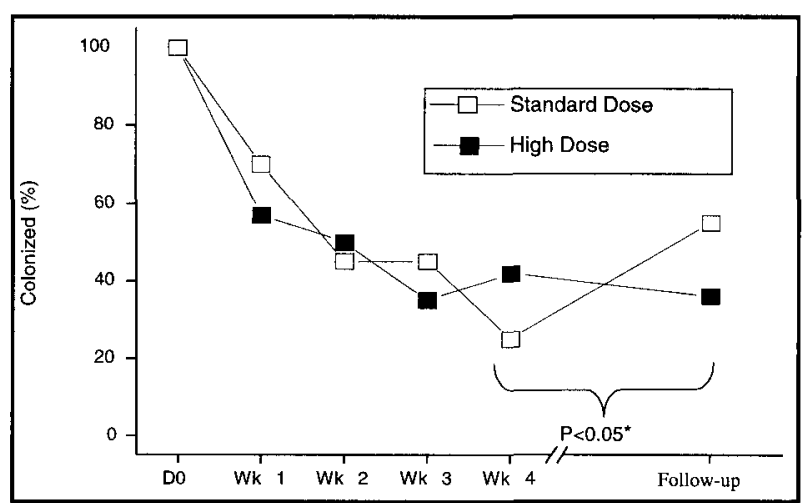

FIGURE. Global (nares, groin, and wound) rate of methicillin-resistant Staphylococcus aureus (MRSA) colonization during and after vancomycin therapy: standard dose $(20 \mathrm{mg} / \mathrm{kg} / \mathrm{d})$ versus high dose $(40 \mathrm{mg} / \mathrm{kg} / \mathrm{d})$. *During the 2-month follow-up period after therapy with a high dose of vancomycin, overall MRSA carriage at any site did not increase, but marginally decreased from $43 \%$ to $36 \%$. Patients treated with a high dose of vancomycin had a higher probability $(P=.048$ ) of remaining free of MRSA during the follow-up period than did patients treated with a standard dose.

regimens for nasal and extranasal MRSA carriage, conflicting data on their efficacy have been reported. ${ }^{18}$ True comparisons among the different studies are difficult because more than 40 different decolonization regimens have been tested in past decades ${ }^{18}$; intranasal application of mupirocin, by far the most effective topical agent, has limited effectiveness in eradicating extranasal MRSA colonization $^{18,19}$; and randomized or nonrandomized prospective studies were performed mostly with noninfected patients in attempts to decrease the incidence of $S$. aureus infections in special risk groups ${ }^{20,21}$ or before orthopedic ${ }^{11}$ or cardiothoracic surgery. ${ }^{22,23}$ When topical decontamination, frequently associated with systemic antimicrobial therapy, was used for controlling endemic or epidemic conditions of MRSA transmission, even more heterogeneous results have been reported ${ }^{2430}$ The discrepancy in the success rates reported in these studies may result not only from the different regimens of antimicrobials used for MRSA decontamination such as co-trimoxazole, rifampin, ciprofloxacin, minocycline, rifampin, and fusidic acid, but also from the highly different clinical and epidemiologic situations of targeted groups. However, these studies did determine that combined regimens of antimicrobial agents were superior to single agents such as rifampin, quinolone, or fusidic acid, with each of these potentially leading to the rapid emergence of antibiotic resistance. ${ }^{31-34}$

This study was facilitated by the well-established MRSA surveillance system in the orthopedic septic unit of our hospital, which involves routine preoperative and postoperative microbiological screening of each patient. To optimize the penetration of vancomycin into bones infected with MRSA and its efficacy, therapy with a higher dose of vancomycin was initiated in our institution in 2001..$^{10,35,36}$ The possible relationship between the duration or amount of vancomycin therapy and the development of resistance 
is still under debate. ${ }^{3740}$ Despite that more than $50 \%$ of patients presenting with VISA received long-term vancomycin treatment ( 29 days to 18 weeks), these isolated cases did not establish a statistically valid relationship between overall vancomycin use and the emergence of glycopeptide resistance. ${ }^{38}$ Because most clinical isolates present as heterogeneous VISA, and thus might represent an intermediate step toward the development of more highly resistant organisms, ${ }^{41}$ we found it appropriate to perform our screening assays at a low concentration $(2 \mu \mathrm{g} / \mathrm{mL})$ of vancomycin. This procedure, added to the Etest with a high inoculum, was rapid and efficient for the detection of trends of emerging vancomycin resistance.

Therapy with a high dose of vancomycin may contribute to the elimination of MRSA carriage without promoting the emergence of glycopeptide resistance. Further studies are warranted to evaluate a larger group of patients during longer follow-up periods.

\section{REFERENCES}

1. Hiramatsu $\mathrm{K}$, Hanaki $\mathrm{H}$, Ino $T$, Yabuta $\mathrm{K}$, Oguri $\mathrm{T}$, Tenover FC. Methicillin-resistant Staphylococcus aureus clinical strain with reduced vancomycin susceptibility. J Antimicrob Chemother 1997;40:135-136.

2. Hiramatsu K, Aritaka N, Hanaki H, et al. Dissemination in Japanese hospitals of strains of Staphylococcus aureus heterogeneously resistant to vancomycin. Lancet 1997;350:1670-1673.

3. Tenover FC, Lancaster MV, Hill BC, et al. Characterization of staphylococci with reduced susceptibilities to vancomycin and other glycopeptides. J Clin Microbiol 1998;36:1020-1027.

4. Sieradzki K, Roberts RB, Haber SW, Tomasz A. The development of vancomycin resistance in a patient with methicillin-resistant Staphylococcus aureus infection. N Engl J Med 1999;340:517-523.

5. Tabaqchali S. Vancomycin-resistant Staphylococcus aureus: apocalypse now? Lancet 1997;350:1644-1645.

6. Hubert SK, Mohammed JM, Fridkin SK, et al. Glycopeptide-intermediate Staphylococcus aureus: evaluation of a novel screening method and results of a survey of selected U.S. hospitals. $J$ Clin Microbiol 1999;37:3590-3593.

7. Pujol M, Peña C, Pallares R, et al. Nosocomial Staphylococcus aureus bacteremia among nasal carriers of methicillin-resistant and methicillin-susceptible strains. Am J Med 1996;100:509-516.

8. Gentry LO. Antibiotic therapy for osteomyelitis. Infect Dis Clin North Am 1990;4:485-499.

9. Lew DP, Waldvogel FA. Osteomyelitis. $N$ Engl J Med 1997;336:999 1007.

10. Bernard L, El-Hajj, Pron B, et al. Outpatient parenteral antimicrobial therapy (OPAT) for the treatment of osteomyelitis: evaluation of efficacy, tolerance and cost. J Clin Pharm Ther 2001;26:445-451.

11. Gernaat-van der Sluis AJ, Hoogenboom-Verdegaal AM, Edixhoven PJ, et al. Prophylactic mupirocin could reduce orthopedic wound infections: 1,044 patients treated with mupirocin compared with 1,260 historical controls. Acta Orthop Scand 1998;69:412-414.

12. Harbarth S, Martin Y, Rohner P, Henry N, Auckenthaler R, Pittet D. Effect of delayed infection control measures on a hospital outbreak of methicillin-resistant Staphylococcus aureus. J Hosp Infect 2000;46:43-49.

13. National Committee for Clinical Laboratory Standards. Performance Standards for Antimicrobial Disk Susceptibility Tests, 4th ed. Wayne, PA: National Committee for Clinical Laboratory Standards; 1990. Approved standard M2-A4.

14. Walsh TR, Bolmström A, Qwärnström A, et al. Evaluation of current methods for detection of staphylococci with reduced susceptibility to glycopeptides. J Clin Microbiol 2001;39:2439-2444.

15. Tenover FC. Implications of vancomycin-resistant Staphylococcus aureus. J Hosp Infect 1999;43(suppl):S3-S7.

16. Wei MQ, Wang FU, Grubb WB. Use of contour-clamped homogeneous electric field (CHEF) electrophoresis to type methicillin-resistant Staphylococcus aureus. J Med Microbiol 1992;36:172-176.

17. Tenover FC, Arbeit RD, Goering RV, et al. Interpreting chromosomal DNA restriction patterns produced by pulsed-field gel electrophoresis: criteria for bacterial strain typing. J Clin Microbiol 1995;33:2233-2239.

18. Boyce JM. MRSA patients: proven methods to treat colonization and infection. J Hosp Infect 2001;48(suppl A):S9-S14.

19. Singh N, Paterson DL, Chang FY, et al, Methicillin-resistant Staphylococcus aureus: the other emerging resistant gram-positive coccus among liver transplant recipients. Clin Infect Dis 2000;30:322-327.

20. Martin JN, Perdreau-Remington F, Kartalija M, et al. A randomized clinical trial of mupirocin in the eradication of Staphylococcus aureus nasal carriage in human immunodeficiency virus disease. $J$ Infect Dis 1999;180:896-899.

21. Kluytmans JA, Manders MJ, van Bommel E, Verbrugh H. Elimination of nasal carriage of Staphylococcus aureus in hemodialysis patients. Infect Control Hosp Epidemiol 1996;17:793-797.

22. VandenBergh MF, Kluytmans JA, van Hout BA, et al. Cost-effectiveness of perioperative mupirocin nasal ointment in cardiothoracic surgery. Infect Control Hosp Epidemiol 1996;17:786-792.

23. Cimochowski GE, Harostock MD, Brown R, et al. Intranasal mupirocin reduces sternal wound infection after open heart surgery in diabetics and nondiabetics. Ann Thorac Surg 2001;71:1572-1578.

24, Muder RR, Boldin M, Brennen C, et al. A controlled trial of rifampicin, minocycline, and rifampicin plus minocycline for eradication of methicillin-resistant Staphylococcus aureus in long-term care patients. I Antimicrob Chemother 1994;34:189-190.

25. Asensio A, Guerrero A, Quereda C, et al. Colonization and infection with methicillin-resistant Staphylococcus aureus: associated factors and eradication. Infect Control Hosp Epidemiol 1996;17:20-28.

26. Darouiche R, Wright C, Hamill R, et al. Eradication of colonization by methicillin-resistant Staphylococcus aureus by using oral minocyclinerifampin and topical mupirocin. Antimicrob Agents Chemother 1991;35:1612-1615.

27. Smith SM, Eng RH, Tecson-Tumang F, Ciprofloxacin therapy for methicillin-resistant Staphylococcus aureus infections or colonizations. Antimicrob Agents Chemother 1989;33:181-184.

28. Parras F, Guerrero MC, Bouza E, et al. Comparative study of mupirocin and oral co-trimoxazole plus topical fusidic acid in eradication of nasal carriage of methicillin-resistant Staphylococcus aureus. Antimicrob Agents Chemother 1995;39:175-179.

29. Ellison RT, Judson FN, Peterson LC, et al. Oral rifampin and trimethoprim/sulfamethoxazole therapy in asymptomatic carriers of methicillin-resistant Staphylococcus aureus infections. West $J$ Med 1984;140:735-740.

30. Roccaforte JS, Bittner MJ, Stumpf CA, Preheim LC. Attempts to eradicate methicillin-resistant Staphylococcus aureus colonization with the use of trimethoprim-sulfamethoxazole, rifampin, and bacitracin. $A m J$ Infect Control 1988;16:141-146.

31. Peterson LR, Quick JN, Jensen B, et al. Emergence of ciprofloxacin resistance in nosocomial methicillin-resistant Staphylococcus aureus isolates: resistance during ciprofloxacin plus rifampin therapy for methicillin-resistant $S$. aureus colonization. Arch Intern Med 1990;150:2151-2155.

32. Chang SC, Hsieh SM, Chen ML, et al. Oral fusidic acid fails to eradicate methicillin-resistant Staphylococcus aureus colonization and results in emergence of fusidic acid-resistant strains. Diagn Microbiol Infect Dis 2000;36:131-136.

33. Mulligan ME, Ruane PJ, Johnston L, et al. Ciprofloxacin for eradication of methicillin-resistant Staphylococcus aureus colonization. Am J Med 1987;82:215-219.

34. Canawati HN, Tuddenham WJ, Sapico FL, et al. Failure of rifampin to eradicate methicillin-resistant Staphylococcus aureus colonization. Clin Ther 1982;4:526-531.

35. Massias L, Dubois C, de Lentdecker P, Brodaty O, Fischler M, Farinotti R. Penetration of vancomycin in uninfected sternal bone. Antimicrob Agents Chemother 1992;36:2539-2541.

36. Kitzes-Cohen R, Farin D, Piva G, et al. Pharmacokinetics of vancomycin administered as prophylaxis before cardiac surgery. Ther Drug Monit 2000;22:661-667.

37. Linares J. The VISA/GISA problem: therapeutic implications. Clin Microbiol Infect 2001;7(suppl 4):8-15.

38. Geisel R, Schmitz FJ, Fluit AC, et al. Emergence, mechanism, and clinical implications of reduced glycopeptide susceptibility in Staphylococcus aureus. Eur J Clin Microbiol Infect Dis 2001;20:685-697.

39. Burnie J, Matthews R, Jiman-Fatami A, Gottardello P, Hodgetts S, D'arcy S. Analysis of 42 cases of septicemia caused by an epidemic strain of methicillin-resistant Staphylococcus aureus: evidence of resistance to vancomycin. Clin Infect Dis 2000;31:684-689.

40. Pina P, Marliere C, Vandenesch F, et al. An outbreak of Staphylococcus aureus strains with reduced susceptibility to glycopeptides in a French general hospital. Clin Infect Dis 2000;31:1306-1308.

41. Hiramatsu K. Vancomycin-resistant Staphylococcus aureus: a new model of antibiotic resistance. Lancet Infect Dis 2001;1:147-155. 